Herz 2013 · 38:327-328

DOI 10.1007/s00059-013-3825-z

Online publiziert: 20. April 2013

(c) Urban \& Vogel 2013

R. Dörr ${ }^{1} \cdot$ H. Thiele ${ }^{2}$

1 Praxisklinik Herz und Gefäße, Akademische Lehrpraxisklinik der TU Dresden, Dresden

${ }^{2}$ Klinik für Innere Medizin/Kardiologie, Universität Leipzig - Herzzentrum, Leipzig

\title{
Diagnostik und Therapie der chronischen Myokardischämie
}

\author{
Wann welche Diagnostik \\ und welche Therapie?
}

Im aktuellen Heft zur Diagnostik und Therapie der chronischen Myokardischämie haben wir versucht, namhafte Experten zu gewinnen, die auf ihrem Gebiet international bekannt sind. Die Kollegen Jacobshagen und Maier aus Göttingen geben einen einleitenden Überblick über die Pathophysiologie der chronischen Myokardischämie, was für die weitere Diagnostik und pathophysiologische Erwägungen zur Therapie elementar ist. Der aktuelle Stand in der Diagnostik der KHK und auch bei der Prognosebeurteilung wird in einem Beitrag der Kollegen von Bardeleben und Tiemann zur Stressechokardiographie, von Hachamovitch zur Myokardszintigraphie und von de Waha und Thiele zur MRT dargestellt. Hierbei wird deutlich, dass in Bezug auf den Ausschluss einer Ischämie oder einer relevanten Koronarstenose für alle bildgebenden Verfahren eine hohe prognostische Relevanz mit einem exzellenten negativ-prädiktiven Wert gezeigt werden konnte. Ebenso entwickeln sich alle Methoden stetig weiter, was es teilweise schwierig macht, die bildgebenden Verfahren miteinander zu vergleichen, da jede publizierte Vergleichsstudie oft bereits nach Abschluss durch die technischen Entwicklungen schon wieder nicht mehr auf dem aktuellen Stand ist. Daher sollte man sich bei der Auswahl des nicht-invasiven Verfahrens sicherlich von den Kosten eines Verfahrens, aber auch von der methodenspezifischen lokalen Experti- se eines diagnostischen Zentrums leiten lassen.

Basierend auf der anatomischen Koronardarstellung, aber auch der aktuell noch nicht im breiten klinischen Alltag einsetzbaren Beurteilung der funktionellen Relevanz von Koronarstenosen mittels CT, die von den Kollegen Schuhbäck und Achenbach federführend erarbeitet wurden, stellen Dörr und Kollegen den aktuellen Stand der sicherlich in der $\mathrm{Zu}$ kunft eine wichtige Rolle einnehmenden Hybridbildgebung dar. Die Kombination aus Anatomie und Funktion ist sicherlich sehr vielversprechend, allerdings aufgrund der bisher noch begrenzten Verfügbarkeit und der ebenfalls noch limitierten Evidenz vorwiegend spezialisierten Zentren zugänglich.

Obwohl die koronare Bypass-Operation und die perkutane Koronarintervention (PCI) etablierte Revaskularisationsverfahren sind, ist die Indikationsstellung bei der chronisch-stabilen KHK eine schwierige Entscheidung. Für eine Revaskularisation aus prognostischer Indikation stellen Ausmaß der Ischämie, linksventrikuläre Pumpfunktion und auch Ausmaß der KHK wesentliche Entscheidungskriterien dar. Andererseits kann auch eine symptomatische Indikation bestehen, wenn die Patienten trotz optimaler medikamentöser Therapie symptomatisch bleiben. Während es für die koronare Bypass-Operation Daten aus randomisierten Studien gibt, dass bei einer enttionellen Flusseserve angewendet wird. 
sprechenden Risikokonstellation die Bypass-Operation die Prognose der Patienten verbessern kann, konnte dies für die PCI derzeit durch randomisierte Studien weiterhin nicht abschließend gezeigt werden. Die aktuelle Datenlage für die Revaskularisation mittels Bypass-Operation oder PCI, aber auch mit einer konservativen optimalen medikamentösen Therapie stellen die federführenden Autoren der COURAGE-Studie [1], Torosoff und Boden, gewohnt kritisch dar. Die derzeitige Datenlage zur Beurteilung der funktionellen Relevanz der KHK, basierend auf der intrakoronaren FFR („fractional flow reserve")-Messung, und die Entscheidung für oder gegen eine Revaskularisation mittels PCI wird durch die federführenden Kollegen der FAME-Studie [2], Tonino und van Nunen aus den Niederlanden, dargelegt. Die FAME-II-Studie bei Patienten mit koronarer Mehrgefäßerkrankung und einer FFR unter 0,80 wurde vorzeitig abgebrochen, weil sich in der Untergruppe mit einer alleinigen optimalen medikamentösen Therapie eine signifikant höhere Rate dringlicher Revaskularisationen im Vergleich zur Untergruppe mit PCI plus optimaler medikamentöser Therapie ergab [3].

Die vielen Diskussionen nach der $\mathrm{Pu}$ blikation dieser FAME-II-Studie zeigen den weiterhin vorhandenen Bedarf an gut geplanten und durchgeführten Studien bei der chronischen Myokardischämie an [4]. Weiterführende Daten zur Beantwortung der Frage, ob die ischämiegesteuerte Revaskularisation mittels PCI zu einer signifikanten Senkung der kardialen Ereignisrate führt, werden von der im Jahr 2012 initiierten ISCHEMIA-Studie [5] erwartet.

Wir hoffen, dass Sie viel Spaß beim Lesen dieser Ausgabe von Herz haben werden.

Rolf Dörr

Holger Thiele

\section{Korrespondenzadresse}

\section{Dr. R. Dörr}

Praxisklinik Herz und Gefäße, Akademische Lehrpraxisklinik der TU Dresden Forststraße 3, 01099 Dresden

doerr@praxisklinik-dresden.de

\section{Prof. Dr. H. Thiele}

Klinik für Innere Medizin/Kardiologie,

Universität Leipzig - Herzzentrum

Strümpellstraße 39, 04289 Leipzig

thielh@medizin.uni-leipzig.de

\section{Literatur}

1. Boden WE, O'Rourke RA, Teo KK et al; COURAGE Trial Research Group (2007) Optimal medical therapy with or without $\mathrm{PCI}$ for stable coronary disease. N Engl J Med 356:1503-1516

2. Tonino PA, De Bruyne B, Pijls NH et al; FAME Study Investigators (2009) Fractional flow reserve versus angiography for guiding percutaneous coronary intervention. N Engl J Med 360:213-224

3. De Bruyne B, Pijls NH, Kalesan B et al; FAME 2 Trial Investigators (2012) Fractional flow reserve-guided $\mathrm{PCl}$ versus medical therapy in stable coronary disease. N Engl J Med 367:991-1001

4. Boden WE (2012) Which is more enduring - FAME or COURAGE? N Engl J Med 367:1059-1061

5. ISCHEMIA Trial: https://www.ischemiatrial.org 